\title{
Utility of Platelet Function Tests: A Recent Review Round Up
}

\author{
Sandeep Sharan ${ }^{1}$ Ajay Gandhi ${ }^{2}$ Poonam Malhotra Kapoor ${ }^{1}$ \\ ${ }^{1}$ Department of Cardiac Anaesthesia, All India Institute of Medical \\ Sciences, New Delhi, India \\ 2Instrumentation Laboratory India Pvt Ltd, Delhi, India \\ Address for correspondence Sandeep Sharan, Department \\ of Cardiac Anaesthesia, All India Institute of Medical Sciences, \\ New Delhi 110029, India (e-mail: sandypmch06@gmail.com).
}

J Card Crit Care TSS 2020;3:24-27

\begin{abstract}
Patients undergoing cardiac surgery are at risk of excessive bleeding and associated complications. Excessive bleeding during and after cardiac surgery has an incidence of $\sim 20 \%$. Massive bleeding and subsequent requirement for blood product administration and mediastinal re-exploration is associated with significant morbidity and mortality. Postoperative, nonsurgical bleeding in cardiac surgical patients is often multifactorial. Platelet dysfunction, excessive fibrinolysis, hypothermia, preoperative anemia, and deficiency of coagulation factors or their dilution are all suggested etiologies of postoperative bleeding. Among these, the most important is thought to be platelet dysfunction, which occurs as a result of the interplay of acquired and pharmacologically induced factors. Patients suffering from coronary artery disease are usually advised to stop antiplatelet medication a few days prior to coronary artery bypass grafting ( $C A B G$ ) to reduce the incidence of postoperative bleeding. However, patients who are still on antiplatelet drugs are at an increased risk of postoperative bleeding. Currently, the transfusion of blood and blood components to manage postoperative

Keywords

- CABG

- recent review round up

- utility of platelet function tests bleeding after CABG remains largely empirical, with considerable variation among institutions. Algorithm-based hemostatic therapy has been shown to be superior to empiric hemostatic therapy that is based on clinical judgment. Hence, there is a need to have objective tests to demonstrate platelet dysfunction before platelet transfusion. Several devices of platelet function tests have been reported in clinical studies to evaluate platelet dysfunction and quantify the need for antiplatelet therapy.
\end{abstract}

\section{Introduction}

Patients undergoing cardiac surgery are at risk of excessive bleeding and associated complications. ${ }^{1}$ Excessive bleeding during and after cardiac surgery has an incidence of $\sim 20 \%{ }^{2}$ Massive bleeding and subsequent requirement for blood product administration and mediastinal re-exploration is associated with significant morbidity and mortality. ${ }^{3}$ Postoperative, nonsurgical bleeding in cardiac surgical patients is often multifactorial. Platelet dysfunction, excessive fibrinolysis, hypothermia, preoperative anemia, and deficiency of coagulation factors or their dilution are all suggested etiologies of postoperative bleeding. ${ }^{2}$ Among these, the most important is thought to be platelet dysfunction, which occurs

published online January 6, 2020
DoI https://doi.org/ 10.1055/s-0039-3402366 ISSN 2457-0206.

as a result of the interplay of acquired and pharmacologically induced factors. ${ }^{4.5}$ Patients suffering from coronary artery disease are usually advised to stop antiplatelet medication a few days prior to coronary artery bypass grafting (CABG) to reduce the incidence of postoperative bleeding. ${ }^{6}$ However, patients who are still on antiplatelet drugs are at an increased risk of postoperative bleeding. ${ }^{7.8}$ Currently, the transfusion of blood and blood components to manage postoperative bleeding after CABG remains largely empirical, with considerable variation among institutions. ${ }^{10}$ Algorithm-based hemostatic therapy has been shown to be superior to empiric hemostatic therapy that is based on clinical judgment. ${ }^{9-12}$ Hence, there is a need to have objective tests to demonstrate platelet dysfunction before platelet transfusion. ${ }^{10}$ Several devices of

Copyright $\odot 2020$ Official Publication of The Simulation Society (TSS), accredited by International Society of Cardiovascular Ultrasound (ISCU).
License terms

(1) (1) $\Theta \circledast$ 
platelet function tests have been reported in clinical studies to evaluate platelet dysfunction and quantify the need for antiplatelet therapy. ${ }^{7,10}$

\section{Innumerable Studies Pave the Way for PFA to Detect Type of Blood Product to Be Transfused}

Platelet dysfunction may contribute to hemostatic alterations in cardiac surgery and can be influenced by preoperative antiplatelet therapy, comorbidities, or the effects of cardiopulmonary bypass itself. ${ }^{13}$ Therefore, perioperative platelet function analysis might have value in prediction of excessive bleeding and transfusion requirements and guiding hemostatic interventions.

Fattorutto et al studied if the platelet function analyzer (PFA-100) could predict blood loss after cardiopulmonary bypass. PFA-100 device failed to predict postoperative bleeding in CABG patients. ${ }^{14}$ Berger et al prospectively studied 50 patients of patients undergoing isolated CABG to determine the relationships among several assays (Verify Now, TEG, AggreGuideTM, Platelet Works, Vasodilator-Stimulated Phosphoprotein Phosphorylation, and Light Transmission Aggregometry) purported to assess platelet function and perioperative bleeding outcomes. There has been so far, very poor correlation among the different methods of assessing platelet function and the utility of any one platelet function parameter of predicting perioperative bleeding. ${ }^{15}$ Whether any of these assays should be used to guide decision making in individual patients is unclear. Treatment with P2Y12 receptor antagonists increases the risk for perioperative bleeding, but there is individual variation in the antiplatelet effect and time to offset of this effect. Malm et al investigated whether preoperative platelet function analyzed using multiplate whole blood aggregometry predicts the risk of bleeding complications in ticagrelor-treated cardiac surgery patients. They concluded that preoperative adenosine diphosphate (ADP)-induced platelet aggregability predicts the risk for severe bleeding complications in ticagrelor-treated cardiac surgery patients. ${ }^{16}$ Della Corte et al studied the possible value of routine aggregometry testing for bleeding prediction following on pump CABG in patients who received preoperative antiplatelet therapy. Aggregometry was analyzed by multiplate analyzer immediately before surgery.

They concluded that aggregometry testing can help in predicting postoperative bleeding in patients undergoing CABG. ${ }^{17}$ Petricevic et al assessed platelet function by two whole blood impedance aggregometers (ROTEM Platelet and Multiplate, TEM International GmbH, Munich, Germany), using three different activators (arachidonic acid, ADP, and thrombin receptor-activating peptide-6), at three perioperative time points (before anesthesia, after aortic declamping, and 5 to 10 minutes after protamine administration). Platelet function was impaired over the time-course in all assays. Both devices provided similar predictability for postoperative chest tube drainage and red blood cell transfusion requirements. ${ }^{18}$
The ROTEM- Platelet is a new module that can be added to the ROTEM. It measures platelet aggregation using impedance aggregometry. During measurement, platelets are activated with different agonists. In the ARATEM test (arachidonic acid thrombo-elastometry) platelets are activated with arachidonic acid, in ADPTEM (adenosine disphosphate thrombo-elastometry) with ADP, and, in TRAPTEM (thrombin activating peptide thrombo-elastometry) with thrombin receptor-activating peptide 6 . Measurement time is 6 minutes, and results are expressed in three different parameters: A6 (amplitude at 6 minutes, in Ohm), MS (maximum slope of the aggregation curve in $\mathrm{Ohm} / \mathrm{min}$ ) and AUC (area under the curve in Ohm.min). A6 describes how well platelets aggregate after selective activation. MS is a measure of the rate of aggregation and describes how quickly platelets aggregate after selective activation. The AUC represents the area under the aggregation curve, from the start of the measurement until 6 minutes of run time. AUC reflects the overall platelet aggregation. ${ }^{19}$

\section{The Standard Laboratory Tests versus Platelet Function Tests}

The standard laboratory tests of the coagulation system (platelet, international normalized ratio, activated partial thromboplastin time) performed before each procedure have a small predictive value ( - Fig. 1). Only thrombocytopenia of $<50 \times 109 / \mathrm{L}$ is associated with significant hemostatic disorders. Despite the fact that the platelet count does not provide any information about their activation potential, it still remains to be the standard in the preoperative bleeding risk assessment in cardiac surgery patients. The activity of platelet receptors and the degree of their blockade are important parameters that should also be assessed in the preoperative setting. Long waiting time for the result of the standard laboratory tests poses also a serious problem especially in the situation of acute hemostatic disruption. The latest European recommendations regarding the management of massive bleeding and coagulopathy include alternative diagnostic methods based mainly on the point-of-care (POC) principle, which provides a quick and a bedside assessment of coagulation disorders.

Vascular hemostasis is followed by platelet hemostasis (activation, adhesion, and aggregation of platelets), which is the prelude to the cascade of enzymatic processes with the final effect of clot formation. A favorable platelet response to vascular injury and blood loss initiates a plasma coagulation cascade. On the other hand, this physiological defense reaction in atherosclerosis patients may cause thrombotic disease, which may result in a myocardial infarction or ischemic stroke. The monitoring of platelet function is used in the diagnosis of acquired and congenital thrombocytopathy as well as in the optimization in the dosage of antiplatelet drugs, preoperative screening of patients qualified for surgery, and to determine the possible need for blood products. Multiplate Analyzer (Roche, F. Hoffmann-La Roche Ltd., Switzerland) is a POC device that allows, in a very short 


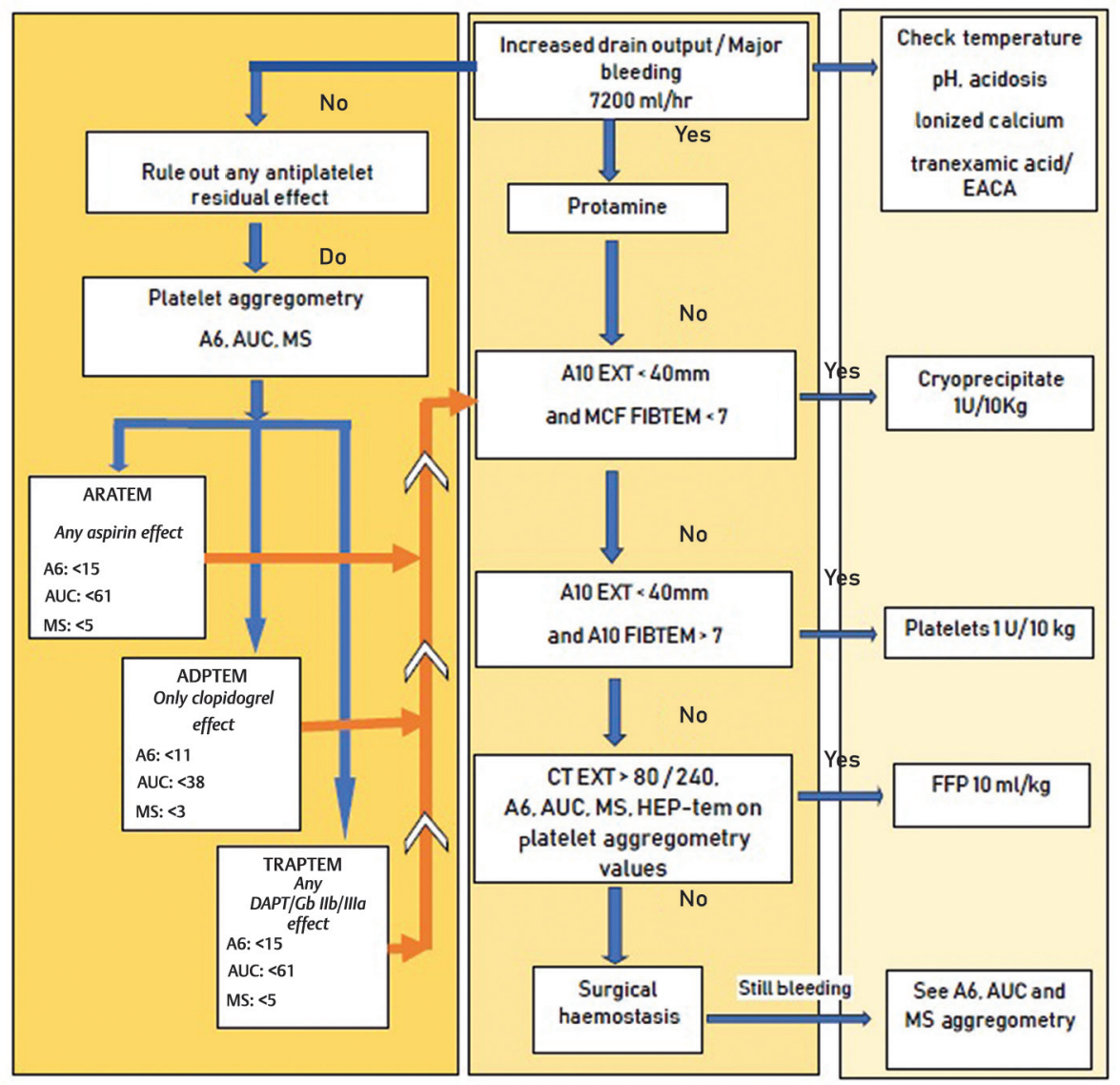

Fig. 1 Algorithm used at AIIMS for testing platelet aggregometry in coronary artery bypass grafting patients. AUC, area under the curve; ARATEM, arachidonic acid thrombo-elastometry; ADPTEM, adenosine disphosphate thrombo-elastometry; EACA, epsilon amino caproic acid; FFP, fresh frozen plasma; HEP, heparin; MS, maximum slope; TRAPTEM, thrombin activating peptide thrombo-elastometry.

time, the bedside assessment of the platelet activity. In patients undergoing cardiac surgery, coagulation diagnosis methods, such as thromboelastometry and the Multiplate Analyzer, are finding a wider and wider application, entering the standards of the safe patient delivery through the procedure.

The POC method for assessing the platelet responses to ADP was included in the Society of Thoracic Surgeons and Society of Cardiovascular Anaesthesiologists Guidelines for Blood Conservation Clinical Practice. ${ }^{20}$ It allows the identification of patients who are insensitive to P2Y12 inhibitors, in particular to clopidogrel. It has a special application in patients qualified for urgent procedures because it allows skipping the recommended waiting period after discontinuation of antiplatelet therapy, to carry out the procedure safely and early enough (Class IIb, Level of Evidence C).

\section{Conclusion}

Early identification of patients at high risk of bleeding, using POC platelet function assessment tests, enables a targeted therapeutic pathway. Due to the variety of factors affecting the activity of platelets, finding a specific cause of this pathology is extremely difficult. According to our study, the correlation between platelet receptor disorders and mild-to-moderate liver and kidney injury has not been demonstrated. However, platelet receptors dysfunction has been shown to be associated with a decreased number of platelets.

\section{Conflict of Interest}

None declared.

\section{References}

1 Karkouti K, Wijeysundera DN, Yau TM, et al. The independent association of massive blood loss with mortality in cardiac surgery. Transfusion 2004;44(10):1453-1462

2 Paparella D, Brister SJ, Buchanan MR. Coagulation disorders of cardiopulmonary bypass: a review. Intensive Care Med 2004;30(10):1873-1881

3 Ranucci M, Baryshnikova E, Castelvecchio S, Pelissero G; Surgical and Clinical Outcome Research (SCORE) Group. Major bleeding, transfusions, and anemia: the deadly triad of cardiac surgery. Ann Thorac Surg 2013;96(2):478-485

4 Rinder CS, Bohnert J, Rinder HM, Mitchell J, Ault K, Hillman R. Platelet activation and aggregation during cardiopulmonary bypass. Anesthesiology 1991;75(3):388-393

5 Holloway DS, Summaria L, Sandesara J, Vagher JP, Alexander JC, Caprini JA. Decreased platelet number and function and increased fibrinolysis contribute to postoperative bleeding in cardiopulmonary bypass patients. Thromb Haemost 1988;59(1):62-67

6 Kozek-Langenecker SA, Afshari A, Albaladejo P, et al. Management of severe perioperative bleeding: guidelines from the European Society of Anaesthesiology. Eur J Anaesthesiol 2013;30(6):270-382 
7 Di Dedda U, Ranucci M, Baryshnikova E, Castelvecchio S; Surgical and Clinical Outcome Research Group. Thienopyridines resistance and recovery of platelet function after discontinuation of thienopyridines in cardiac surgery patients. Eur J Cardiothorac Surg 2014;45(1):165-170

8 Reece MJ, Klein AA, Salviz EA, et al. Near-patient platelet function testing in patients undergoing coronary artery surgery: a pilot study. Anaesthesia 2011;66(2):97-103

9 Murphy GJ, Reeves BC, Rogers CA, Rizvi SI, Culliford L, Angelini GD. Increased mortality, postoperative morbidity, and cost after red blood cell transfusion in patients having cardiac surgery. Circulation 2007;116(22):2544-2552

10 Weber CF, Görlinger K, Meininger D, et al. Point-of-care testing: a prospective, randomized clinical trial of efficacy in coagulopathic cardiac surgery patients. Anesthesiology 2012;117(3):531-547

11 Ranucci M, Baryshnikova E, Soro G, Ballotta A, De Benedetti D, Conti D; Surgical and Clinical Outcome Research (SCORE) Group. Multiple electrode whole-blood aggregometry and bleeding in cardiac surgery patients receiving thienopyridines. Ann Thorac Surg 2011;91(1):123-129

12 Cannon CP, Mehta SR, Aranki SF. Balancing the benefit and risk of oral antiplatelet agents in coronary artery bypass surgery. Ann Thorac Surg 2005;80(2):768-779

13 Fattorutto M, Pradier O, Schmartz D, Ickx B, Barvais L. Does the platelet function analyser (PFA-100) predict blood loss after cardiopulmonary bypass? Br J Anaesth 2003;90(5):692-693
14 Berger PB, Kirchner HL, Wagner ES, et al. Does preoperative platelet function predict bleeding in patients undergoing off pump coronary artery bypass surgery? J Interv Cardiol 2015;28(3):223-232

15 Malm CJ, Hansson EC, Åkesson J, et al. Preoperative platelet function predicts perioperative bleeding complications in ticagrelor-treated cardiac surgery patients: a prospective observational study. Br J Anaesth 2016;117(3):309-315

16 Della Corte A, Bancone C, Spadafora A, et al. Postoperative bleeding in coronary artery bypass patients on double antiplatelet therapy: predictive value of preoperative aggregometry. Eur J Cardiothorac Surg 2017;52(5):901-908

17 Petricevic M, Konosic S, Biocina B, et al. Bleeding risk assessment in patients undergoing elective cardiac surgery using ROTEM(®) platelet and Multiplate( $\left({ }^{\circledR}\right)$ impedance aggregometry. Anaesthesia 2016;71(6):636-647

18 Bhardwaj V, Kapoor PM. Platelet aggregometry interpretation using ROTEM - PART - II. Ann Card Anaesth 2016;19(4):584-586

19 Nissen PH, Skipper MT, Hvas AM. Whole blood platelet aggregation determined by the ROTEM platelet equipment; reference intervals and stability. Platelets 2019;1595562:1-6

20 Lambert MP. Platelets in liver and renal disease. Hematology (Am Soc Hematol Educ Program) 2016;2016(1):251-255 